\title{
Cooperative Model for Idle Traffic Optimisation
}

\author{
Hrvoje Baričević, Jasmin Ćelić, Siniša Vilke
}

The growth trend and the trend of population migration into city centres, as well as the continuous increase in the number of motor vehicles cause traffic jams and congestions in most major cities in the world. In order to eliminate the problems caused by the circling in search of available parking space, i.e. by the congestion, different methods and approaches are implemented. First of all, these are the appropriate strategies and policies that are made in order to regulate the existing situation and to demotivate the arrival of part of the population in the city centre, which does not represent a necessity. Another way to eliminate the above problem, which is the most emphasized in this paper, is represented by the intelligent parking systems. Intelligent parking systems are used to guide the driver to the available parking space and to provide information on the characteristics of it. Normally, they are also used for parking reservation and payment, but in most cases they were related exclusively to closed parking lots and garages. With the technological progress and the development of intelligent sensors it has become possible even to control street parking spaces, which is especially emphasized in this paper. Also, an advanced model of guidance to available parking spaces using a cooperative approach is proposed. The simulation model presenting the advantages of this system compared to the classical approach, which are also highlighted in this paper, is developed.

\section{KEY WORDS}

$\sim$ Intelligent Transportation Systems (ITS)

$\sim$ Cooperative approach

$\sim$ Advanced Transport Information Systems

$\sim$ Guidance and Navigation Systems

$\sim$ Routes searching

$\sim$ Parking lots in ports

University of Rijeka, Faculty of Maritime Studies Rijeka, Rijeka, Croatia e-mail: hrvoje@pfri.hr

\section{INTRODUCTION}

The development of urban centres and the increasing number of vehicles on the roads around the world, and increasing demand for parking spaces in city centres seriously affects the quality of traffic. Searching for free parking places increases the traffic volume which cannot be adequately met by the existing capacity of the transport network. This problem is particularly expressed in large harbours, especially during the tourist season (Baričević, 2001). Consequently, this may affect traffic congestion and thus reduce the demand for travel in the desired area. Elasticity, as one of the characteristics of this approach for resolving city centre congestion, enables precise alignment to the target group of users in relation to space and time. The main goal of the system for information and referral to the parking spaces (Parking Guidance and Information, PGI) is to reduce traffic caused by searching for a suitable free parking space in city centres and large parking area (Krpan et al., 2007). At the entrances and exits of the parking lots or on individual parking spaces sensors or devices for counting vehicles to allow the collection of data on the number of occupied parking spaces are usually installed. Sensors used for this purpose are usually induction loops, infrared, ultrasound and magnetic sensors (Zhou and Li, 2014), laser technology, etc. The development of information and communication technologies and continued efforts to reduce the dimensions of electronic components and circuits enabled the installation of a microcontroller, a wireless transceiver and a power supply, together with certain sensor, in the traffic infrastructure (Jeffrey et al., 2012; Ji et al., 2014; Geng and Cassandras, 2012). These intelligent sensors provide partially or fully processed information to its users in the form of messages which usually, amongst the other data, contain the position of available parking spaces and information about possible transport routes to the same, and may provide other 
useful information such as zones, total number of available parking spaces in the parking lot, cost of parking and etc. All of this technology does not provide any guarantee that intelligent parking system will work efficiently in all possible conditions (Zanella et al., 2014).

\section{APPLICATION OF ITS FOR LOCATING VEHICLES AND NAVIGATION}

One of the basic requirements for quality performance of intelligent parking systems, especially in terms of on-street parking, is the information about the vehicle position. Automatic Vehicle Location (Automatic Vehicle Location, AVL) is a range of technologies that include technology positioning, mapping and communications. AVL technology is rarely used as a standalone application, but is an integral part of many ITS services such as route guides, transit passenger information, fleet management of commercial vehicles, computer-assisted forwarding, congestion detection and return of stolen vehicles. The basic components of the AVL and navigation technologies are:

- location technology and positioning systems,

- maps,

- geographic Information Systems (GIS) and map-matching,

- route guidance and detecting route

- display and distribution of information.

A special approach for determining the position is in the use of mobile devices (Figure 1). The main advantage of this approach is the ability to use the existing infrastructure, which significantly reduces the costs of the establishment of such services. Mobile telephony has already allocated frequency band and a large user base that is required to support such an idea.

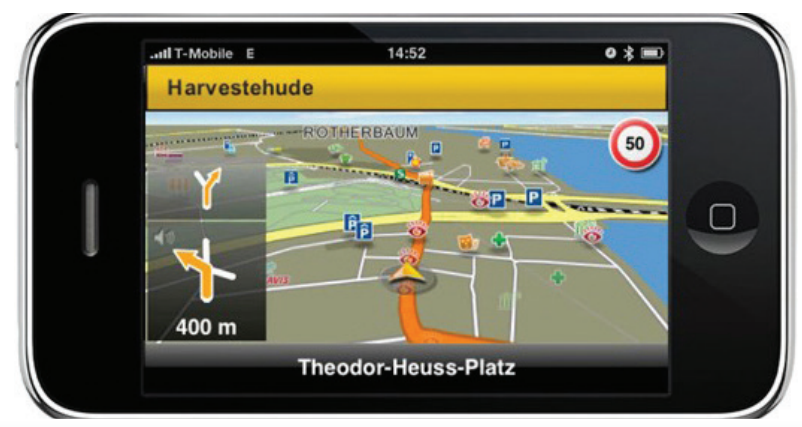

Figure 1.

Navigation system NAVIGON on connecting mobile devices.

For the purpose of positioning and navigation different charts are used, usually raster or vector maps. Raster maps are basically images that are suitable for showing details such as height differences using topographical indications. Vector maps are drawings made up of points, lines and polygons stored in the database in the form of $\mathrm{x}$ and $\mathrm{y}$ coordinates. Vector maps show much less graphic details from the raster map. Raster maps have a number of disadvantages which are, regardless of their benefits, almost useless in most AVL applications. They require far more memory than vector maps; they need much more time for rendering on the device display; and are fixed relative to the orientation because when turned text will rotate and going to be showed upside. Also, regardless of the increase or decrease in the chart it will show the same level of detail, and the parts of the text may become completely illegible.

\section{ADVANCED MODEL REFERENCES AVAILABLE ON THE VEHICLE PARKING SPACES}

Simulators of traffic are an essential tool for the study and research of traffic. Microsimulation software solutions are able to model the movement of individual vehicles traveling a predetermined road network. Mathematical models are essentially an integral part of simulators, and provide a realistic view of the behaviour of drivers in the road network. Unlike deterministic models, stochastic approach and better resolution of microscopic simulation software solutions provide a better view of the behaviour of drivers and vehicles in the transport network, especially when it comes to complex traffic problems such as the impact of incidents or parking on the traffic entirely. Models of road traffic basically consist of transported entities, road vehicles and the road network. Each of these factors is distinguished by specific characteristics that should be determined as part of the simulation model in order to achieve realistic conditions in which the proposed advanced system should operate. For the purpose of this study a conceptual model of the system that refers to available parking spaces by using a cooperative approach has been made. The proposed system is in contrast to similar existing solutions based primarily on cooperation between on-board unit (OBU) and roadside unit (RSU). The data provided by the Advanced Traveller Information Systems (ATIS) is used only in cases where it is necessary (no vehicles in the communication range, there is no need for historical data, etc.), in order to achieve effective information sharing. Information from the sensors, which are part of the transport infrastructure, are processed on the spot using a microcontroller and forwarded directly to the system in a nearby vehicle, which then forwards the information to other vehicles in the series until it finds a person of interest. So, sensors of the road unit and the vehicle have a direct ability to process the data and sort of intelligence to be considered as microcomputer units. A vehicle equipped with the appropriate equipment on the arrival in the area of communication range of RSU starts receiving the available information and transfers them to other vehicles around it. At the same time this information, depending on the communication capabilities, forwards itself to 


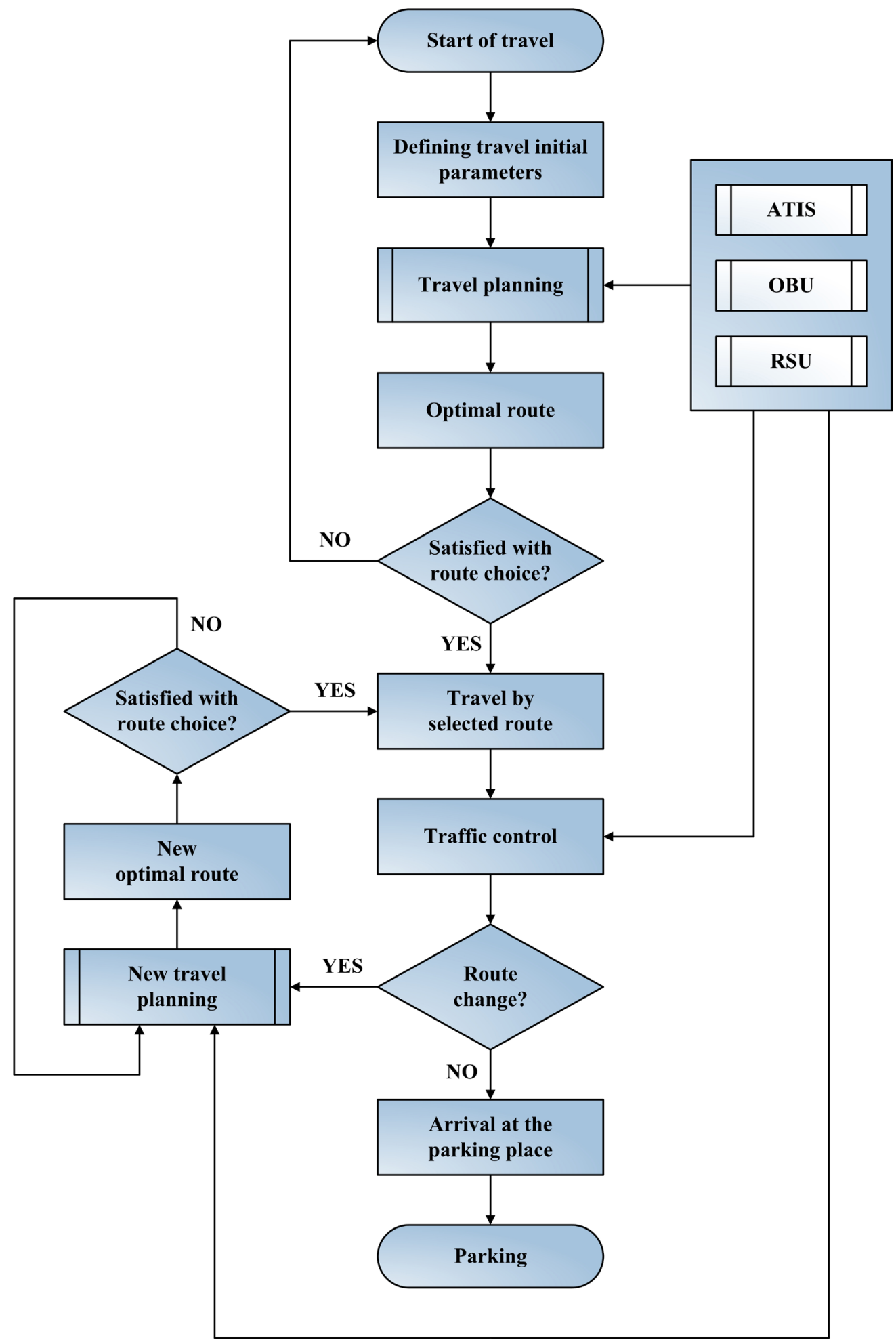

Figure 2.

Flowchart travel, search of available parking space and parking with a cooperative approach. 
the ATIS. Based on the above cooperation between vehicles and RSU the information about the availability of parking spaces is transferred. When entering the street or arriving in the port zone where the parking spaces are, vehicles know in advance where to park, thus reducing the need for unnecessary circling in search of available parking space.

\subsection{The Calculation of Travel Time and Total Cost}

Traffic situation and thus travel times are changing during the period that takes time to assign the parking lot. The total simulation time is divided into smaller intervals to easily evaluate considered travel time. It should not be less than 5 minutes and no more than half the time interval set by the origin-destination (OD) matrix. During the simulation process the travel time for each edge of the network (time between two nodes) is measured separately. Every vehicle after leaving a certain edge reports about time spent, and then calculates the average of all travel time spent at the edge which then presents the resulting travel time. Special attention is given to vehicles that are retained on an edge longer than the duration of the evaluation period because it is a sign of the presence of congestion. This is the report about duration of outages. The measured travel time is not used for the purposes of search and selection of route in the same iteration, but its influence on the next one.

The development of models and simulations was achieved by the usage of the software solution named VISSIM. Namely, simulation program VISSIM allows the use of not only the travel time from the iterations that immediately preceded the current but also all other early iterations, in two ways (PTV VISSIM 6 User Manual, 2014):

1. Exponential smoothing - the user determines the smoothing factor that calculates the impact of previous iterations so that the oldest iteration have the least impact,

2. Method of Successive Averages (MSA) - calculates the arithmetic medium of all iterations, and on the basis that determines the weight factor. The higher the number of iterations that are considered in analysis, the less the influence of each next iteration.

Smoothed travel time is calculated as s result from the set of values, as a weighted sum of the old iteration and current iteration. The results of smoothed value represent travel time which is expected in the next iteration. Travel time for particular edge measured in the time interval of the one iteration is exponentially smoothed before making a decision about selection of the route, in a following way (Barcelo, 2010):

$T_{a}^{n, K}=(1-a) \cdot T_{a}^{n-1, k}+a \cdot T O_{a}^{n, k}$ significance:

$K$-index of evaluation intervals during the period of simulation,

$n$-index of iteration allocation

$a$ - edge index

$\mathrm{TO}_{a}{ }^{n, K}$-measured travel time on the edge $a$ for period $k$ in iteration $n$

$T_{a}^{n, K}$ - expected travel time on the edge of $a$ for period $k$ in iteration $n$

$a$ - smoothing factor.

Estimated travel time using MSA is calculated as follows:

$T_{i}^{n, K}=\left(1-\frac{1}{N+n}\right) \cdot T_{i}^{n-1, K}+\frac{1}{N+n} \cdot T O_{i}^{n, K}$

meaning:

$N$ - user-determined value,

$K$ - index of evaluation of the intervals during the simulation

$n$ - index of iteration allocation

$i$ - edge index

$T O_{i}^{n, k}$ - measured travel time on the edge $i$ for a period $k$ in the iteration $n$,

$T_{i}^{n, K}$ - expected travel time on the edge $i$ and for the period $k$ in the iteration $n$,

$\frac{1}{n+N}$ - variable factor that depends on smoothing parameter $N$ and iteration allocation.

Travel time is not the only factor for influencing the choice of route. The function of general costs is a linear combination of travel time, travel length and the finances, such as toll payment. Unlike the time needed to travel these factors are independent of the traffic situation and are not determined for the simulation. Components of the cost can be determined separately for each class of vehicle that the user had generated earlier. According to these three factors, the overall cost $C$ for each edge is calculated as a weighted sum of:

$C=a \cdot t+\beta \cdot s+\gamma \cdot C_{f}+\Sigma C_{n 2}$

meaning:

$a, \beta, \gamma$ - Factors which may, if necessary, determine the different behaviour of the driver in the choice of routes,

$s$ - determined by the geometry of the link,

$C_{f}$ - sum of all costs of links that form an edge. Cost of the link is the product

of its length in kilometres and the cost per kilometre length specified in its parameters. 
This cost includes the value of the parameter surcharge 1 $C_{n 2}$-additional cost link (surcharge 2 ) that is not loaded with factor $\gamma$.

\subsection{Search and Selection of Suitable Routes}

The route is a series of edges which describes the path through the network. Routes begin and end at the parking spaces. The general cost of the route is determined by the overall cost of its all edges (PTV VISSIM 6 User Manual, 2014):

$$
C_{R}=\sum_{a \in R} C_{a}
$$

meaning:

$C$ - general costs,

$R$ - route.

Between the starting point and the parking lot, there is usually more than one possible path, so simulation program VISSIM's model is used to help the driver in his choice of route. Within the dynamic assignment of VISSIM, it weights the number of available routes and carries out the selection. Route selection is a special case of discrete choice because the probability of selection of alternatives must be calculated for a given set of discrete alternatives. Using the dynamic assignment the set of available routes can be displayed within origin-destination set. There is no efficient algorithm in finding the best of several routes, but there is a possibility of finding the right one (Van der Waerden, 2012). For every OD pair in dynamic iteration the best route is required, which solves the afore mentioned problem, because traffic situations change travel time from iteration to iteration until the certain criteria is satisfied. This will be a way to find a different "best" route that VISSIM will save to a file with .weg extension so that they could be available for future iterations.

The criterion for the selection of the best routes is general expense. Due to the fact that the weighting coefficient overheads depend on vehicle type, different types of paths will be selected for different types of vehicles. In the first iteration, there is no travel time information from previous iterations and, therefore, instead of using the length of travel time, the length of the route in meters is used. A set of known ways will be used more if drivers are somehow encouraged to try new routes. For that purpose, the following iterations of edges, on which the vehicle did not pass, fictitious travel time of 0.1 second will be added in order to increase their attractiveness during searching for a route.
However, this process can lead to useless routes joining the group which is generated. Useless routes are those routes that replace any existing route of the known route, with link of much greater length, and they are considered obvious detours. The length of a series link that will be qualified as a bypass is determined by the user. With dynamic assignment drivers are choosing the route at the time of leaving the source parking lot. One of the basic assumptions when choosing the route in VISSIM is that not all drivers use the best route, but will use all known pathways in a way that the larger share of the traffic will be distributed in better way. Quality of route is evaluated by overheads, which are contrary to the utility, which is part of the theory of discrete decision. Therefore, utility is determined as the reciprocal of overheads (PTV VISSIM 6 User Manual, 2014):

$U_{j}=\frac{1}{C_{j}}$

meaning:

$U_{j}$ - usefulness of travel time $j$,

$C_{j}$ - overheads of travel time $j$.

$A$ - function that is most often used for choice of routes is Logit function (Ben-Akiva et al., 1984):

$p\left(R_{j}\right)=\frac{e^{\mu U_{j}}}{\sum e^{\mu U_{j}}}$

meaning:

$U_{j}$ - usefulness of route $j$,

$\mathrm{p}\left(R_{j}\right)$ - probability of selected route $j$,

$\mu$ - parameter of model sensitivity $(>0)$.

A small value of the parameter sensitivity will result with distribution without larger influence of the utility while the greater value will result in fact that almost every driver will choose the best route. If the Logit function, which is translational irreversible, is applied with the previously mentioned cost function, the model will be assigned the same importance with smaller differences between travel time for smaller values as with the relatively large (e.g. a lot of significant differences in the travel times between 11 and 8 minutes will be identified with almost negligible difference trips of 116 and 113 minutes). In order to approximate the actual assessment and distribute traffic demand to all known routes as OD pairs, VISSIM uses Kirchhoff distribution formula (PTV VISSIM 6 User Manual, 2014): 
$p\left(R_{j}\right)=\frac{U_{j}^{k}}{\sum_{i} U^{k}}$

meaning:

$U_{i}$ - usefulness of route $j$,

$p\left(R_{j}\right)$ - probability of choosing the route $j$,

$k$ - parameter of model.

In this form of the utility the distribution ratio is determined, and it does not represent the absolute difference utility. This means that in the above mentioned case there will be little difference in the allocation of traffic for the paths whose travel times are 116 and 113 minutes, but it will take a lot more traffic to the path whose travel time is 8 minutes compared to the path whose travel time lasts 11 minutes. Kirchhoff distribution formula can be expressed with Logit function if the utility function is displayed in the logarithmic form:

$p\left(R_{j}\right)=\frac{U_{j}^{k}}{\sum_{i} U_{i}^{k}}=\frac{e^{k \cdot \log U_{j}}}{\sum_{i} e^{k \cdot \log U_{j}}}=\frac{e^{-k \cdot \log C_{j}}}{\sum_{i} e^{-k \cdot \log C_{j}}}$

where $C_{j}$ is overall cost of the route $j$.

For the deviation distribution in overlapping route VISSIM offers expansion of route model with factor identity. Based on the current situation in the simulation model and pre-set criteria the parking space for vehicles entering the modelled transport network is dynamically determined. In the case where one of the vehicle without route guidance (RG) occupies a parking place designated for the vehicle with the RG, RG vehicle will select new available parking space and calculate the appropriate route to the same using the same algorithms as in the time of entry into the transportation network simulation model.

\section{CONCLUSION}

Intelligent parking system includes various subsystems, and thus in its implementation inevitably include knowledge from the domain of ITS. Cooperative approach offers quick access to information for users, where information is given as needed without necessarily direct contact with advanced information systems. Vehicles and sensors in the infrastructure, which now actually represent a sort of microcomputers, are able to analyse and process information and forward them to each other for the purpose of better management. This resulted in the proposition of the advanced model of the system using cooperative approach, with the emphasis on the synergistic effect of the optimal route selection and the corresponding available parking space. The proposed advanced system model which refers to the available parking spaces should also encourage the creation of new strategies and policies for parking, which would reduce the costs for city authorities, entrepreneurs and business companies, developers and users, and improve the quality of parking services and achieve substantial benefits for the whole community. It should speed up the process of development of future systems that use similar technology and provide appropriate information to traffic engineers, designers and planners for finding a parking lot and become a solid support for the development of intelligent cities. This is of particular interest in tourist destinations (passenger harbours) in Croatia, where there is an evident peak traffic demand in the time of tourist season.

\section{REFERENCES}

Baričević, H., (2001), Tehnologije kopnenog prometa, Rijeka: Pomorski fakultet u Rijeci and Glosa d.d.

Barcelo, J., (2010), Fundamentals of Traffic Simulations, vol. 145., New York: Springer. Ben-Akiva, M. E., Bergman, M. J., Daly, A. J. and Ramaswamy, R., (1984), Modelling Inter Urban Route Choice Behavior, Ninth International Symposium on Transportation and Traffic Theory, VNU Science Press, pp. 299-330.

Geng, Y. and Cassandras, G. C., (2012), A New 'Smart Parking' System Infrastructure and Implementation, Proceedings of EWGT2012 - $15^{\text {th }}$ Meeting of the EURO Working Group on Transportation, Paris, France, September 2012, pp. 1278-1287., http://dx.doi.org/10.1016/j.sbspro.2012.09.842

Jeffrey, J., Patil, R. G., Narahari, S. K. K., Didagi, Y., Bapat, J. and Dias, D., (2012), Smart Parking System Using Wireless Sensor Networks", $6^{\text {th }}$ International Conference on Sensor Technologies and Applications SENSORCOMM 2012, August 19 - 24, Rome, Italy pp. 306-311.

Ji, Z., Ganchev, I., O’Droma, M., Zhao, L. and Zhang, X., (2014), A Cloud-Based Car Parking Middleware for loT-Based Smart Cities: Design and Implementation, Sensors, 14, pp. 22372-22393.,

http://dx.doi.org/10.3390/s141222372

Krpan, Lj., Baričević, H. and Frka, D., (2007), Directional Parking Garage System- Pilot Project of Application Within the City of Rijeka ZIRP - Transportni lanci i razvitak prometa, Zagreb, Croatia, April 15.

PTV VISSIM 6 User Manual, (2014), Karlsruhe, Germany: Planung Transport Verkehr AG.

Van der Waerden, P., (2012), Pamela, a Parking Analysis Model for Predicting Effects in Local Areas, PhD Thesis, Eindhoven, Netherland: Technische Universiteit Eindhoven, Faculteit Bouwkunda.

Zanella, A., Bui, N., Castellani, A., Vangelista, L. and Zorzi, M., (2014), Internet of Things for Smart Cityes, IEEE Internet of Things Journal, 1(1), pp. 22-32., http://dx.doi.org/10.1109/JIOT.2014.2306328

Zhou, F. and Li, Q., (2014), Parking Guidance System Based on ZigBee and Geomagnetic Sensor Technology, $13^{\text {th }}$ International Symposium on Distributed Computing and Applications to Business, Engineering and Science, Xian Ning, China, November 24-27, pp. 268-271.,

http://dx.doi.org/10.1109/DCABES.2014.58 